\title{
Steam Generator Mock-up for Assessment of Inservice Inspection Technology
}

by

D. S. Kupperman and S. Bakhtiari Argonne National Laboratory

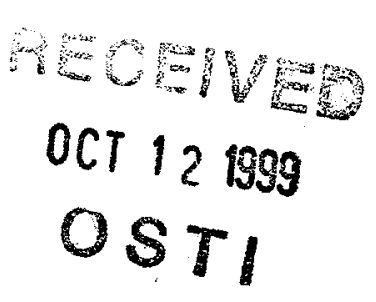

U. S. Nuclear Regulatory Commission

\author{
J. Muscara
}

July 1999

\begin{abstract}
The submitted manuscript has been coauthored by a contractor of the U.S. Government under contract No. W-31-109-ENG38. Accordingly, the U.S. Government retains a nonexclusive, royalty-free license to publish or reproduce the published form of this contribution, or allow others to do so, for U.S. Government purposes.
\end{abstract}

To be presented at SM iRT-15 $11^{\text {th }}$ Post Conference Seminar 2 - Current Issues in the Evolution of Risk Informed and Performance Based Integrity Engineering, University of Tokyo, Tokyo, Japan, August 24, 1999.

*Work supported by the Office of Nuclear Regulatory Research, U. S. Nuclear Regulatory Commission. 


\section{DISCLAIMER}

This report was prepared as an account of work sponsored by an agency of the United States Government. Neither the United States Government nor any agency thereof, nor any of their employees, make any warranty, express or implied, or assumes any legal liability or responsibility for the accuracy, completeness, or usefuiness of any information, apparatus, product, or process disclosed, or represents that its use would not infringe privately owned rights. Reference herein to any specific commercial product, process, or service by trade name, trademark, manufacturer, or otherwise does not necessarily constitute or imply its endorsement, recommendation, or favoring by the United States Government or any agency thereof. The views and opinions of authors expressed herein do not necessarily state or reflect those of the United States Government or any agency thereof. 


\section{DISCLAIMER}

\section{Portions of this document may be illegible in electronic image products. Images are produced from the best available original document.}




\title{
Steam Generator Mock-up for Assessment of Inservice Inspection Technology
}

\author{
D. S. Kupperman and S. Bakhtiari, Argonne National Laboratory \\ J. Muscara, U. S. Nuclear Regulatory Commission \\ SMiRT-15 $11^{\text {th }}$ Post Conference Seminar 2 \\ Current Issues in the Evolution of Risk Informed and \\ Performance Based Integrity Engineering
}

\begin{abstract}
A steam generator mock-up has been assembled for round-robin studies of the effectiveness of currently practiced inservice inspection (ISI) technology for detection of current-day flaws. The mock-up will also be used to evaluate emerging inspection technologies. The 3.66-m (12ft.)-tall mock-up contains 400 tube openings, each consisting of 9 test sections that can be used to simulate current-day field-induced flaws and artifacts. Included in the mock-up are simulations of tube support plate (TSP) intersections and the tube sheet (TS). Cracks are present at the TSP, TS, and in the free span sections of the mock-up. For initial evaluation of the round-robin results, various eddy current methods, as well as multivariate models for data analysis techniques, are being used to estimate the depth and length of defects in the mock-up. To ensure that the round-robin is carried out with procedures as close as possible to those implemented in the field, input was obtained from industry experts on the protocol and procedures to be used for the exercise. One initial assembly of the mock-up with a limited number of flaws and artifact has been completed and tested. A second completed configuration with additional flaw and artifacts simulations will be used for the round-robin.
\end{abstract}

\section{INTRODUCTION}

Over the last several years, the U.S. Nuclear Regulatory Commission has been developing new regulatory guidance for steam generator (SG) tube integrity. Some of this guidance, as well as current industry practices, recommends condition monitoring and operational assessments following the ISI of SG tubes. In condition monitoring, the as-found condition of the tubes is evaluated to determine whether tube integrity performance criteria were met during the last operating cycle. Operational assessment is used to demonstrate that the performance criteria for SG tube integrity will continue to be met until the next scheduled ISI. Quantitative information on probability of detection (POD) and sizing accuracy of the techniques used for ISI of current- 
day flaws is needed to conduct these assessments. Detailed information on inspection reliability will permit estimation of the true state of the generator tubes after ISI by including the flaws that were missed because of imperfect POD. Similarly, knowledge of sizing accuracy will permit corrections in the flaw sizes obtained from ISI.

Eddy-current (EC) inspection techniques are the primary means of ISI for assessing the condition of the SG tubes. Theoretically, there is a relationship between the depth of a defect into the tube wall and the EC signal response. In practice, however, the relationship between signal voltage or phase angle and defect depth is influenced by many other variables, including tube material properties, tube geometry, degradation morphology, human factors, and EC data analysis and acquisition practices.

One of the tasks in the NRC-sponsored Steam Generator Tube Integrity Program at Argonne National Laboratory (ANL) is to evaluate and quantify the reliability of current, advanced, and emerging technology used for ISI of SG tubes with current-day flaws. This program is a part of an international cooperative effort on SG tube integrity research.

An approach to establishing the reliability of current ISI methods could be to carry out round-robin (RR) exercises in the field on either operating steam generators or generators removed from service. However, access to such facilities for this purpose is generally not possible. Such work would be prohibitively expensive, and validation of results would be difficult or impossible. Thus the problem is how to acquire realistic POD and sizing accuracy data. The solution chosen here was to develop an SG tube bundle mock-up that simulates the key features of an SG so that the inspection results from the mock-up would be close to those from an operating SG. The mock-up was constructed to provide nondestructive examination (NDE) signals, flaws, and conditions that are representative of an SG in service.

Because the flaws in the test specimens must be representative of actual flaws that develop in service, considerable effort was expended in preparing realistic flaws and verifying that their EC signals and morphologies are representative of those from operating steam generators. Stress corrosion cracks of different orientations have been incorporated at various locations in the mock-up. Artifacts and support structures that may affect the EC signals were included.

POD and sizing capability will be evaluated by analysis of results from RR inspections carried out on the mock-up. The RR will include EC and ultrasonic testing (UT) methods. Data acquisition and analysis will be performed by commercial teams, and results will be reported for different procedures and techniques.

Laboratory NDE methods such as EC and UT are being evaluated as means to characterize the defects in the mock-up tubes so that the "true state" can be estimated before destructive examination of the test sections is carried out. Multivariate regression analysis and neuralnetwork algorithms are being applied to multiparameter EC data. This effort has led to estimates 
of depth and length of the various tube bundle defects. In addition, an NDE Task Group has been formed to provide input to the RR protocol and inspection procedures.

\section{MOCK-UP DESIGN}

The mock-up tube bundle contains hundreds of 0.3-m (12-in.)-long, $22.2 \mathrm{~mm}$ (7/8-in.)diameter Alloy 600 test sections with various forms of degradation and artifacts. Most of the degraded test sections were produced at ANL, though some test sections are from Pacific Northwest National Laboratory (PNNL), Westinghouse, Equipos Nucleares, SA (ENSA) in Spain, and the Program for the Inspection of Steel Components (PISC).

Figure 1 is a schematic diagram of the mock-up tube bundle, which consists of 400 tube openings, each with 9 levels, for a total of 3600 test sections. The levels are separated by polypropylene plates and the test sections are mechanically fitted into these plates. There is a 0.91-m. (36-in.) runout for the top of the bundle. The lowest level is a TS simulation. The test sections in this level are mechanically expanded onto a 6-in.-long carbon steel collar, leaving a roll transition halfway up the test section. The steel collar is then split and removed from the expanded tube, and the tube is cracked by exposing it to a chemical solution. A new steel collar is expanded by heating and slipped over the cracked tube. This process produces test sections with realistic flaws and EC signals. Rolling of the test section before and adding a different collar after the crack is grown avoids unrealistic deformation of the cracks and nontypical EC signals. Axial and circumferential outer diameter (OD) and inner diameter (ID) stress corrosion cracks (SCC) are located in various roll transition zones. In addition to the TS, there are three 3/4-in.-thick carbon steel, drilled-hole TSP simulations, and five free-span levels in the mockup. Axial ODSCC, both planar and segmented, and cracks in dents with varying morphologies are present at the TSP locations. Cracks in the remaining five-free span levels are primarily axial ODSCC, both planar and segmented. Other types of flaws such as IGA and wear are found in the tube bundle but in small numbers. The specific number and distribution of flaws of various types is not given to maintain the confidentiality of the RR exercise. Magnetite-filled epoxy markers are placed on the ends of all test sections to provide a reference for angular location of flaws when collecting data with a rotating pancake coil (RPC) or array probe. The assembled tube bundle is placed on a platform as shown in Fig. 2.

\section{ARTIFACTS}

Besides the cracks and support structures, other conditions that can affect the flaw signal are incorporated in the mock-up. A significant effort has been made to reproduce the EC signals and distortions from artifacts found in the field. These conditions include roll transitions discussed previously, magnetite, sludge, and spherically and elliptically shaped dents with various combinations of cracks around the dents (denting tools were provided by Framatome Technologies, Inc.). The magnetite is simulated primarily by use of magnetic tape in the TSP 


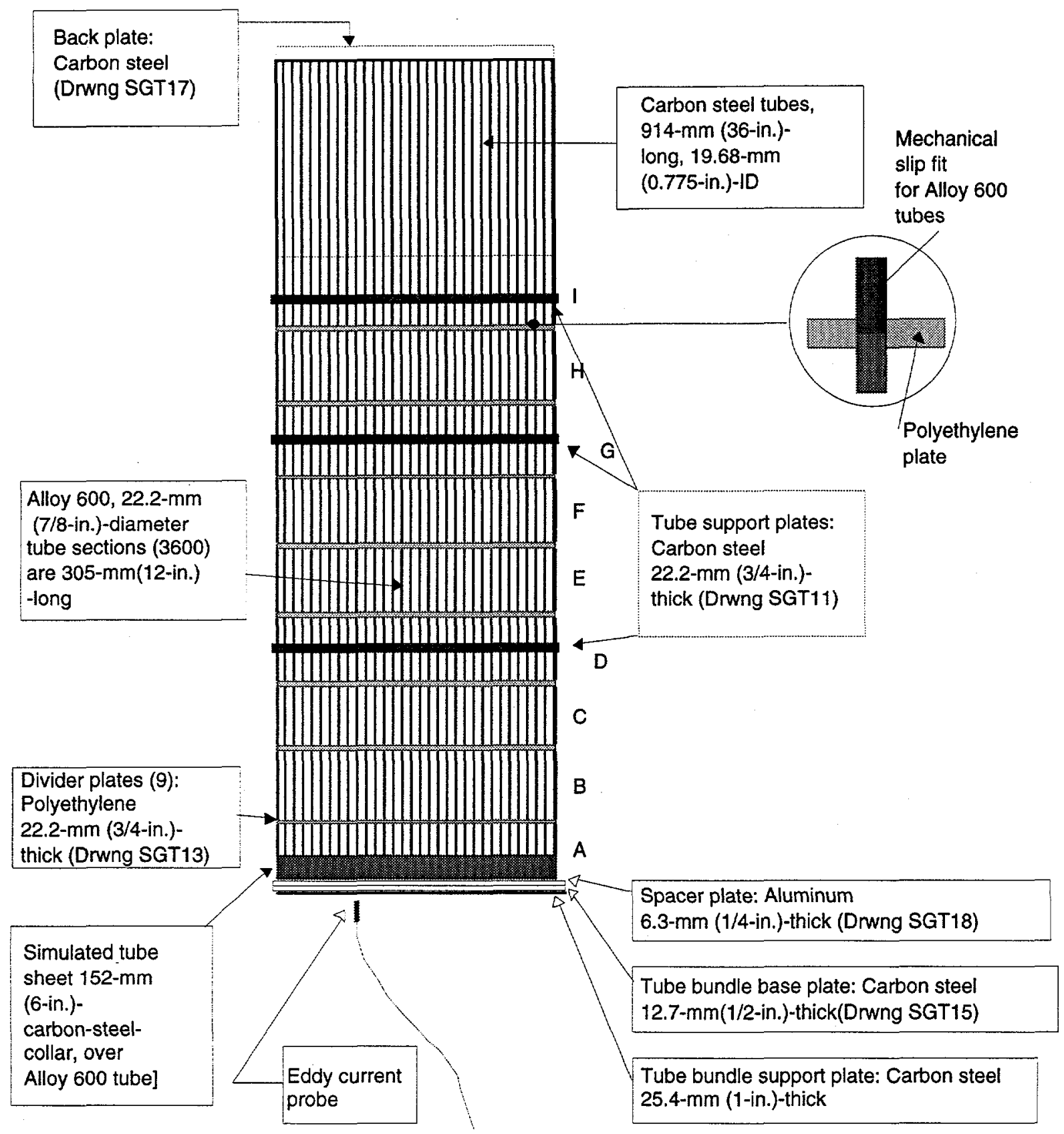

Fig. 1. Schematic diagram of 3.66-m (12-ft.)-high mock-up tube bundle. 


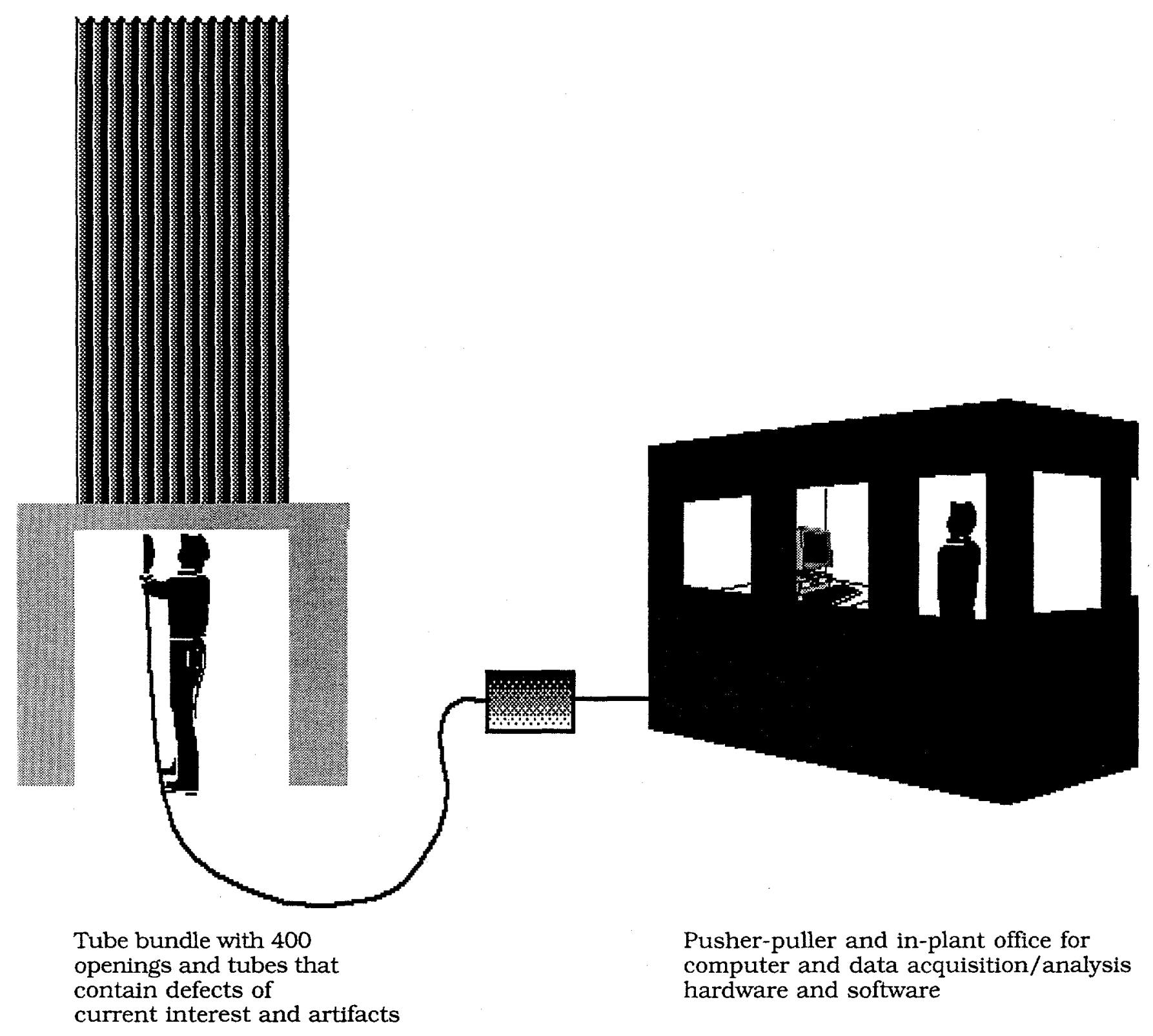

Fig. 2. Schematic diagram of steam generator mock-up inspection facility.

crevice and magnetite mixed with a varnish applied to some free-span test sections. Sludge is a mix of magnetite, copper, and epoxy applied to the top of the tube sheet (and above the TSP in some cases). 


\section{EVALUATION OF NDE TECHINIQUES FOR CHARACTERIZING FLAWS IN THE MOCK-UP}

Knowledge of the "true state" of the flaws in the mock-up is needed to estimate the POD and sizing accuracy of various techniques following the RR exercise. Obtaining the true state of flaws without destroying test sections is necessary to maintain the mock-up for studies after the initial RR is completed. An effort was therefore made to establish the best NDE methods for obtaining good flaw characterization information for the types of flaws in the mock-up. Twenty clean test sections (without interfering signals) with laboratory-grown cracks were examined by a variety of NDE techniques. The test sections were then destructively analyzed. While these 20 test sections are a small subset of the mock-up test sections, the NDE results from them provided guidance for selecting the NDE methods for characterizing all of the test sections. The 20 test sections were characterized by using phase analysis of Zetec + Point ${ }^{\mathrm{TM}}$ probe data, multivariate regression analysis, multiparameter analysis of EC data with neural network linear and nonlinear algorithms, conventional ultrasonic pulse-echo techniques, high-frequency ultrasonic diffraction, ultrasonic Lamb waves, and acoustic microscopy. Outer-diameter cracks were also examined with dye penetrants. While some of the ultrasonic results will be useful for a limited number of test sections in estimating crack depths, the primary method of characterizing the cracks will be multivariate analysis of EC data. An example of the capability of multivariate analysis for characterizing cracks is shown in Fig. 3. Here, the EC depth estimates for the 10 OD cracks (axial and circumferential) of the 20-test-section set are compared with actual depths from destructive analysis. In general, sizing is accurate within about $10 \%$, except for one significant outlier.

\section{NDE ROUND ROBIN}

In preparation for carrying out the RR, a map of the locations and types of flaws was developed for the tube bundle. Also, all data from laboratory NDE of the test sections will be assessed to provide, for each test section, "true-state" baseline data such as flaw depth, length, morphology, and appropriate three-letter code (i.e., MAI for multiple axial indication, SAI for single axial indication, SCI for single circumferential indication, VOL for volumetric indication, etc.). The characterization data will provide the basis for initial estimates of POD and sizing accuracy from the RR test results. The initial estimates will be updated and validated through destructive examination of selected mock-up test sections. The key objective of the RR studies of the mock-up is to establish the POD for flaws of current-day interest as a function of the flaw size, type, and location for the types of equipment, procedures, and personnel used for ISI of SG tubes in the United States. Inspection and data analysis for the mock-up will therefore mimic the inspection process conducted on operating steam generators. The mock-up will be treated as a real steam generator owned by a utility, with ANL designated as the owner. 


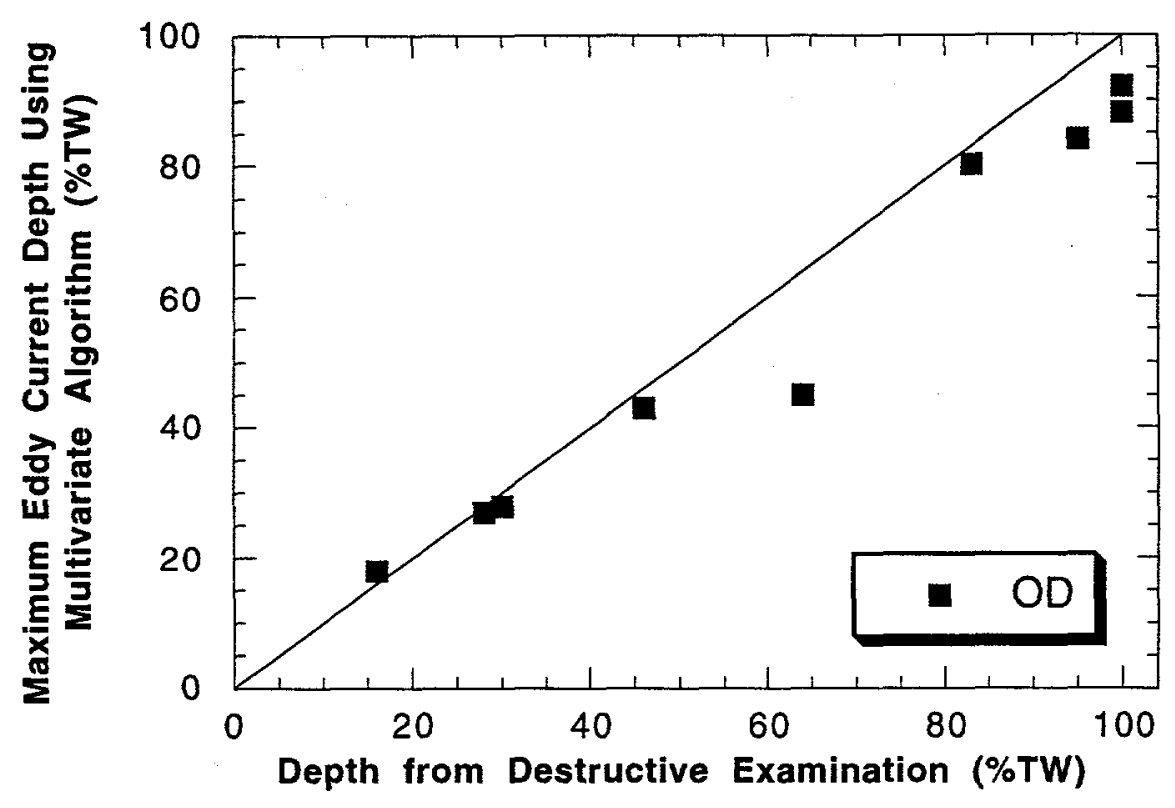

Fig. 3. Maximum eddy current depth as percent throughwall (\%TW) using a pancake coil and multivariate algorithm for 10 OD cracks from the 20-test-section set (axial and circumferential), vs. destructive analysis result provided by PNNL.

An NDE Task Group has been established to provide input to the program. It comprises industry experts and practitioners who were nominated by the participants in the cooperative program. A major role for the Task Group was providing input on the protocol, procedures, and methods used by industry for ISI of SG tubes. Using this input, ANL then developed the protocol, documentation, and requirements for "Inservice Inspection" of the mock-up.

A degradation assessment of the mock-up has been conducted, and qualified techniques and procedures to be used for inspection have been selected. Where more than one qualified technique is available for a given flaw type and location, an effort was made to include the most commonly used techniques for inspection of the mock-up. Examination Technique Specification Sheets and procedures have been identified in the documentation for the mock-up inspection. Eddy current signals from test sections of the mock-up have been reviewed with several members of the NDE Task Group to ensure that the flaw signals and conditions are typical of those found in real SGs. Also, metallographic examinations from specimens typical of those in the mock-up have been reviewed with industry experts to ensure that the crack morphologies in the mock-up specimens are typical of those found in the field. Another indication that the cracks grown at ANL are representative of field cracks comes from a comparison of EC signals from tubes removed from service with those of the ANL samples. Figure 4 shows bobbin coil voltage as a 
function of phase angle for a number of axial ODSCC at tube support plates of tubes removed from the retired SGs at the McGuire 1 nuclear power plant, along with those from the ANLgrown axial ODSCC. The plot shows comparable flaw responses between the two.

Further, the essential variables for the test section flaws and conditions in the mock-up are being reviewed to ensure that detection and sizing capabilities developed in accordance with Appendix H of the EPRI Steam Generator Examination Guidelines, Revision 5, are applicable to site-specific (mock-up-specific) conditions. The RR exercises will use several qualified teams from ISI vendors. Inspection data will be acquired with the MIZ30 and the TC6700 data acquisition systems, which are most commonly used in the United States. Eddynet 98 and ANSER software will be used for data analysis. Approximately ten qualified data analysis teams will be engaged to analyze the mock-up data. Each team will consist of three qualified data analysts: a primary, a secondary and a resolution analyst. Before the analysts begin evaluating the mockup data, they will undergo site-specific (mock-up) training and performance demonstration testing. The procedures, requirements, and evaluations discussed above have been described in various protocol documents to be used for ISI of the mock-up, following a process similar to that done by utility owners before ISI of their operating steam generators. Inspection of the mock-up began in June 1999.

In addition to their use in determining inspection reliability of currently practiced techniques, the inspection results will be used for evaluating the detectability of flaws with and without artifacts, detectability and signal response from notches versus cracks, and accuracy of flaw profiles (flaw depth is a function of flaw length) from the EC data of selected flaws. Further, the mock-up will be available until June 2000 for other RRs and studies (based on international interest) for evaluation of other inspection techniques such as ultrasonics, emerging and advanced technologies, and other EC methods and techniques not included in the initial RR.

\section{SUMMARY}

Nuclear power plants are required to carry out periodic ISIs of SG tubing, to perform condition monitoring and operational assessment, and to repair or remove from service all tubes with degradation exceeding the tube repair limits. EC inspection techniques are the primary means for assessing the condition of the SG tubes. To quantify the reliability of EC techniques, an SG mock-up has been assembled ANL for round-robin studies. The probability of detection and sizing accuracy of currently practiced ISI technologies for current-day flaws will be established. The mock-up will also be used to evaluate emerging inspection technologies. The 3.66-m (12-ft.)-tall mock-up contains 400 tube openings, each consisting of 9 test sections, that are being used to simulate current day flaws and artifacts. An estimate of the "true state" flaw length and depth has been established for the mock-up flaws using advanced EC data analysis techniques. This information will be used for the initial evaluation of the round-robin results. 


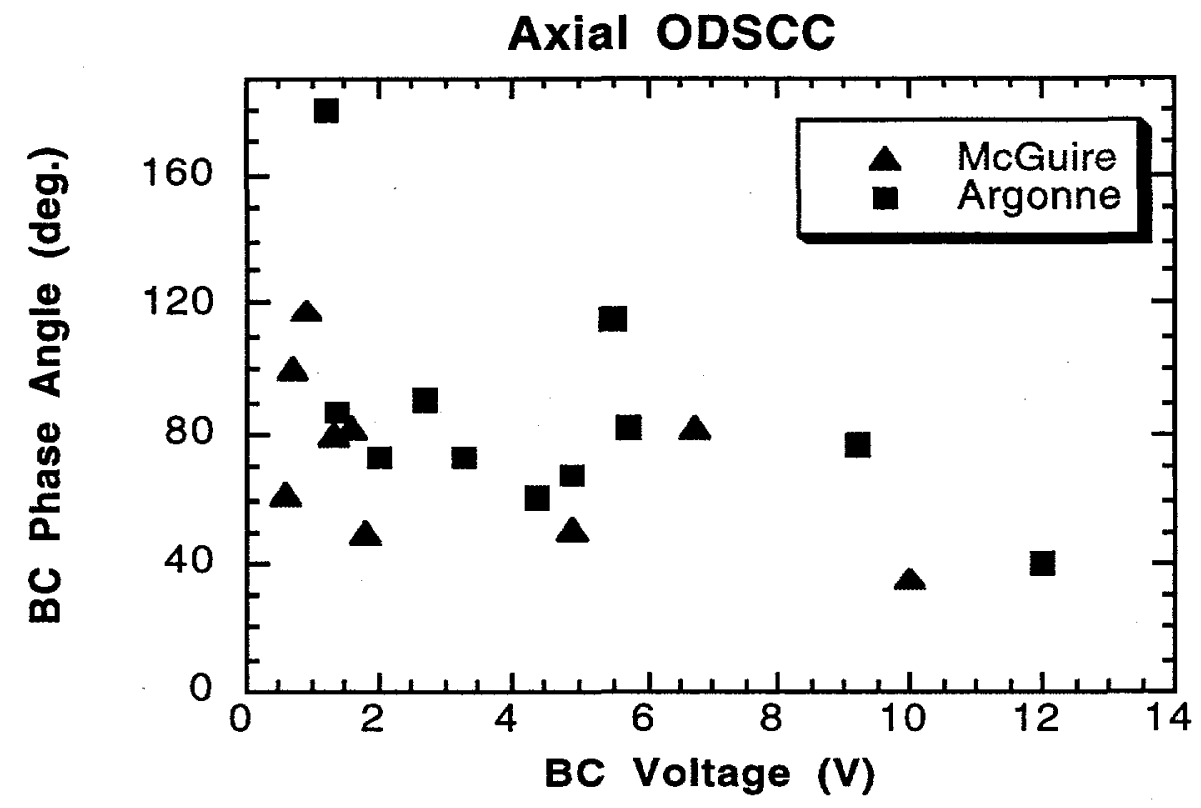

Fig. 4. Comparison of McGuire steam generator SG-D bobbin coil voltages and phase angles from axial ODSCC at tube support plates to voltages and phase from ANL-generated lab-grown axial ODSCC.

The mock-up is being treated as a real generator, and the inspection and data analysis for the initial round-robin mimics the ISI process conducted on operating SGs in the United States. Input from industry experts was obtained to ensure that the mock-up flaws and conditions are realistic and that the inspection protocol simulates industry practice. Inspection of the mock-up for evaluating currently practiced techniques began in June 1999. The mock-up will be available for evaluating other NDE methods and advanced techniques until June 2000. 